East African Medical Journal Vol. 86 No. 11 November 2009

LIFE THREATENING SPINAL SHOCK AND COMPLETE NEUROLOGICAL RECOVERY FOLLOWING MINOR SPINAL CORD TRAUMA IN A PATIENT WITH PRE-EXISTING CERVICAL CANAL STENOSIS: CASE REPORT V. Mung'ayi, MBChB, MMed (Anaes.), FICM, Assistant Professor, Department of Anaesthesia, M. M. Qureshi, MBChB, MMed (Surg), FCS-ECSA, FRCS (Edin) (Surg. Neurol.), Senior Lecturer, Section of Neurosurgery, and J. Bugo, MBChB, Postgraduate Resident, Department of Anaesthesia, Aga Khan University, Hospital, Nairobi, P.O. Box 30270 - 00100 , Nairobi, Kenya

Request for reprints to: Prof. V. Mung'ayi, Department of Anaesthesia, Aga Khan University, Hospital, Nairobi, P.O. Box 30270 - 00100, Nairobi, Kenya

\title{
LIFE THREATENING SPINAL SHOCK AND COMPLETE NEUROLOGICAL RECOVERY FOLLOWING MINOR SPINAL CORD TRAUMA IN A PATIENT WITH PRE-EXISTING CERVICAL CANAL STENOSIS: CASE REPORT
}

\author{
V. MUNG'AYI, M. M. QURESHI and J. BUGO
}

\begin{abstract}
SUMMARY
Mild to moderate trauma to the spinal cord that is complicated by existing cervical canal stenosis or spondylosis can be a life threatening event. It is against this background that we present a 41 year old male with cervical spinal stenosis who developed marked quadriparesis and respiratory embarrassment following collision with a colleague. He was admitted to the intensive care unit, electively sedated, paralysed and ventilated for a period of 48 hours. During the initial 24 hours of admission, he received methylprednisolone as per the National Acute Spinal Cord Injury Studies (NASCIS) protocol. Forty five hours later he had regained full neurological and respiratory function, allowing extubation and subsequent discharge from the intensive care unit. While patients with cervical spinal cord trauma in a setting of cervical canal stenosis or spondylosis can deteriorate dramatically, early recognition of this condition and prompt management using methylprednisolone, cervical stabilisation and ventilatory support during the initial window of opportunity will enable them make a full recovery.
\end{abstract}

\section{INTRODUCTION}

Spinal shock is a state of transient physiologic (rather than anatomic) reflex depression of cord function below the level of injury, with associated loss of all sensorimotor functions. An initial increase in blood pressure due to the release of catecholamines, followed by hypotension, is noted. Flaccid paralysis, including that of the bowel and bladder, is observed, and sometimes sustained priapism develops. These symptoms tend to last several hours to days until the reflex arcs below the level of the injury begin to function again (eg, bulbocavernosus reflex, muscle stretch reflex) (1). We report a patient who developed spinal shock following minimal trauma, during a game of football.

\section{CASE REPORT}

The patient was a 41 year old male. On the day of admission the patient was playing football and accidentally collided with a colleague and fell over. $\mathrm{He}$ did not loose consciousness but was unable to move his upper and lower limbs and he stated that he had pain in his arms and neck. Vital signs recorded by the first aid team were, heart rate $88 / \mathrm{min}$, respiratory rate- $20 / \mathrm{min}$, and blood pressure $118 / 82 \mathrm{mmHg}$.

At the scene of his injury he was evaluated by members of the flying doctor's team and was given ketamine and midazolam for pain and sedation, a hard cervical collar fitted and was then strapped on to a vacuum mattress and airlifted to our hospital.

At the emergency room, his examination revealed an anxious male, alert, oriented and coherent. He was aware of what had caused his injury and was obeying commands. He was mildly dyspnoeic with flaring of the alae nasii. The tone in upper and lower limbs was reduced. Power was grade 2 in the upper limbs and grade 0 in the lower limbs.

Sensation was intact to soft touch, pin prick and joint position. Examination of the chest, abdomen and cardiovascular system was normal. Pulse was $82 / \mathrm{min}$, respiratory rate $12 / \mathrm{min}$, SP02 $97 \%$ on oxygen via nasal prongs at $4 \mathrm{~L} / \mathrm{min}$. BP $135 / 87 \mathrm{mmHg}$. An emergent $\mathrm{CT}$ scan of the head was normal. He was admitted to theintensive care unit(ICU) and electively intubated, sedated, paralysed and ventilated for a period of 48 hours. Methylprednisolone was given 
as per the National Acute Spinal Cord Injury Studies (NASCIS) protocol for a period of 24 hours. He was also managed as per ICU patient care protocols. A subsequent MRI of his cervical and thoracic spine done 24 hours after symptom onset showed multilevel cervical disc osteophyte complexes causing spinal canal stenosis. A high signal was seen in the cord at C5-C6 level suggestive of oedema.

The spinal canal was narrowed with an anteroposterior distance at the level C5-C6 of 9.4 $\mathrm{mm}$ (normal $>13 \mathrm{~mm}$ for the cervical spine) (Figure 1 and 2).

\section{Figure 1}

Sagittal MRI cervical spine

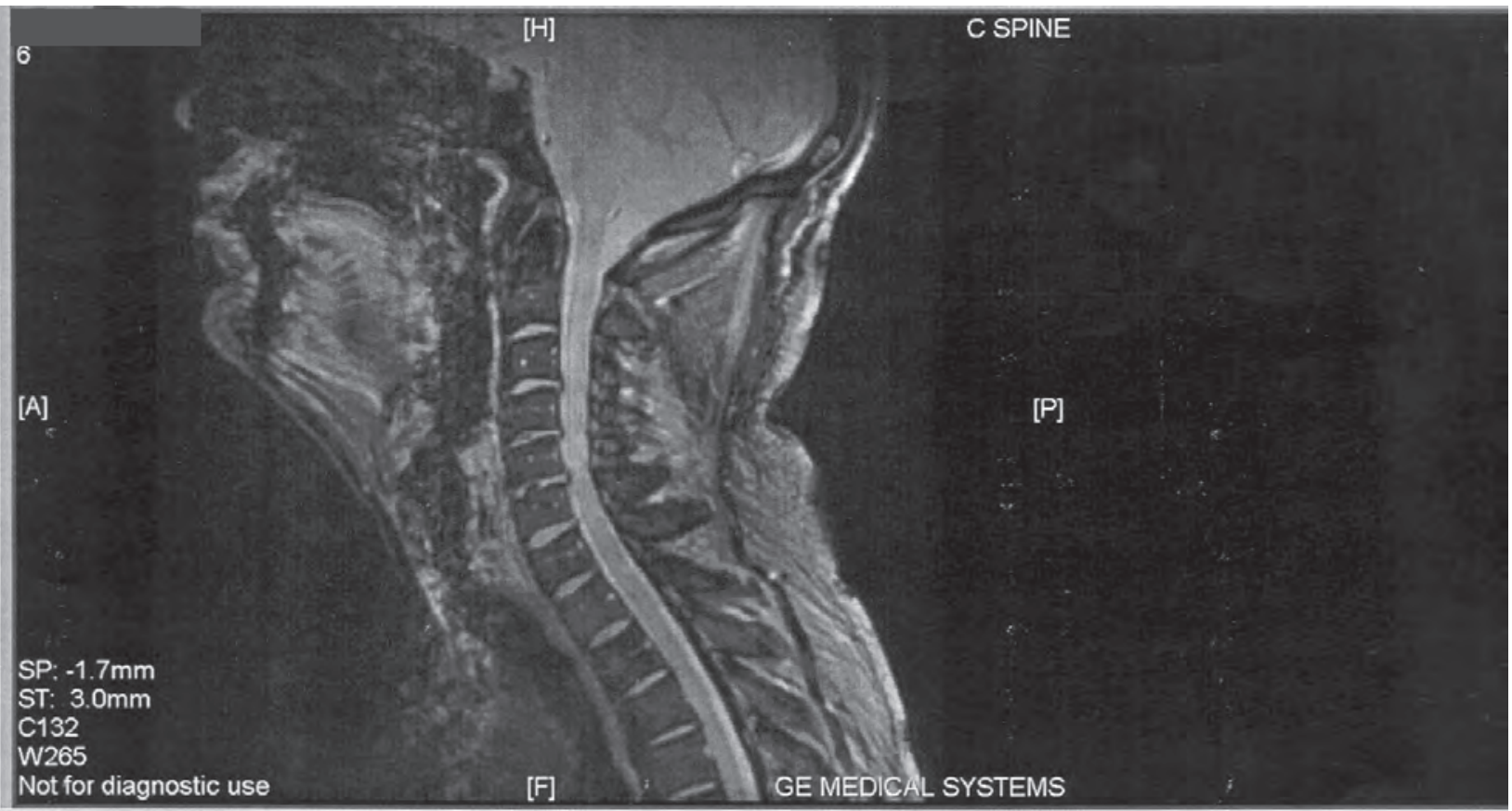

Figure 2

(a)

Axial MRI cervical spine

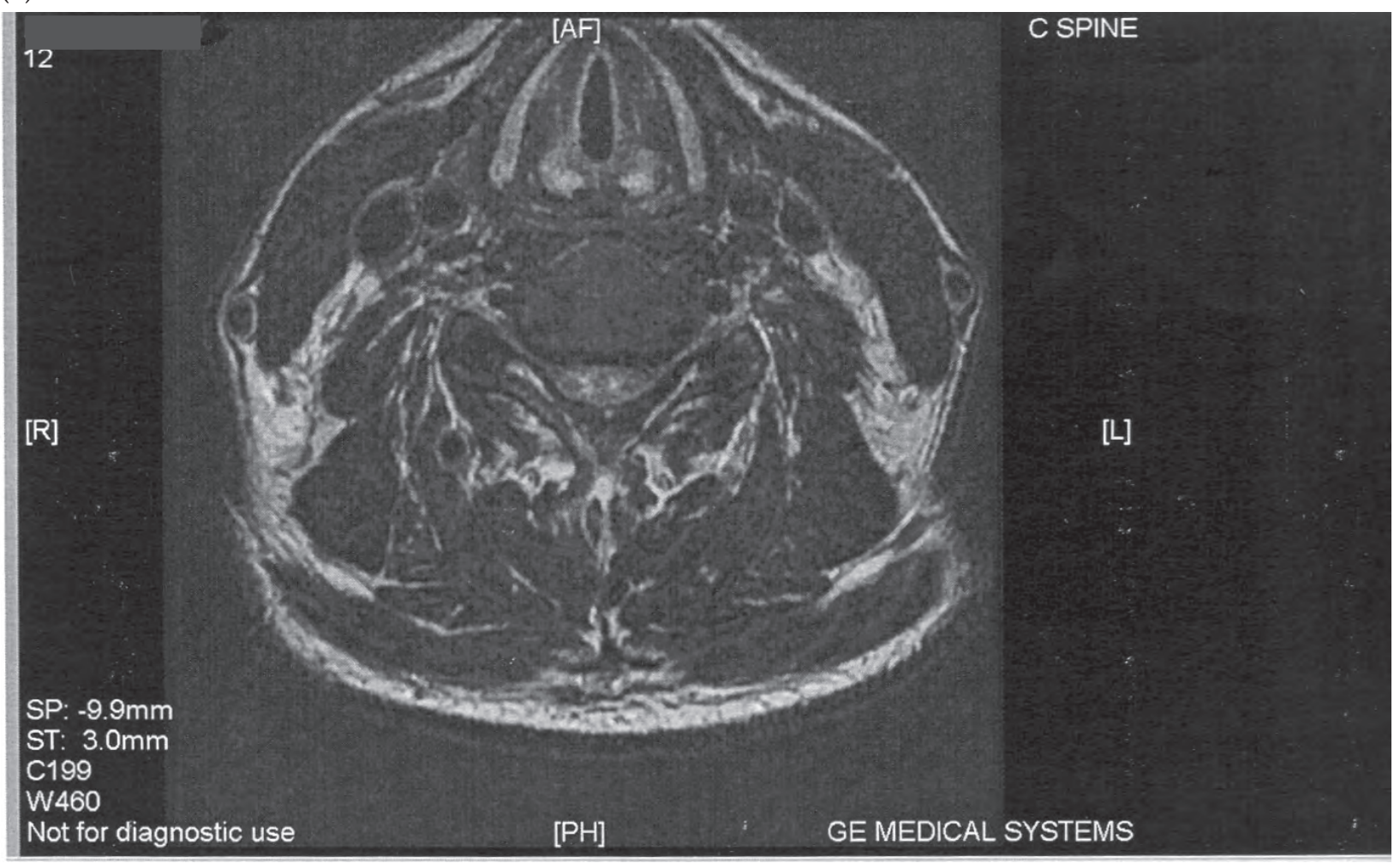


(b)

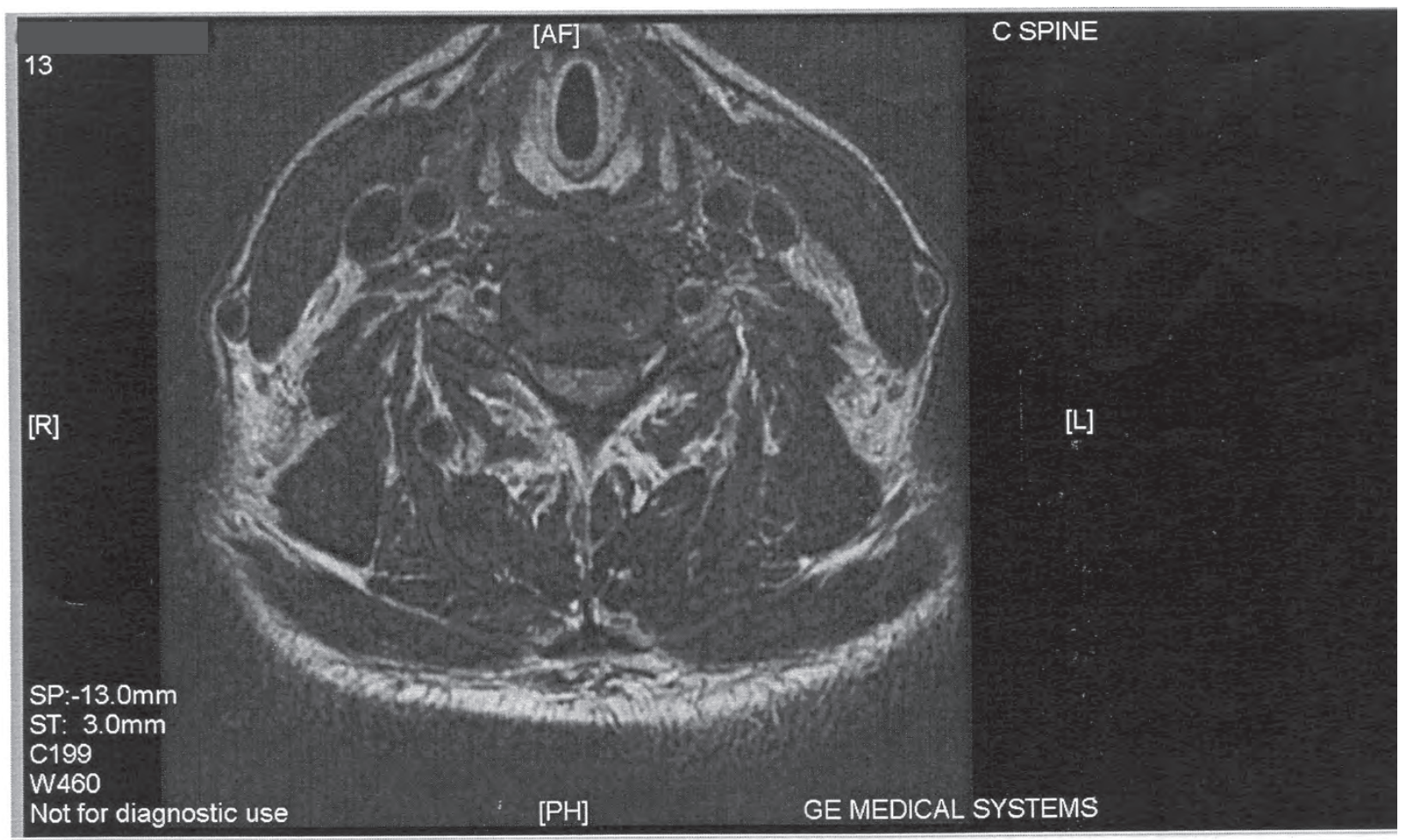

He showed a gradual improvement in respiratory function. The rest of his systemic examination findings remained normal and he was extubated 45 hours from time of intubation and discharged from the ICU. No further clinical episodes occurred during admission. During his recuperation, he provided a history of a road accident ten years earlier which necessitated wearing a neck collar for a few days. Neurological examination at time of discharge revealed a mild impairment of sensation to pinprick in his right index finger. The rest of his neurological examination was entirely normal. He was discharged from hospital on the eleventh day following admission.

\section{DISCUSSION}

Spinal shock is a potentially dangerous complication of trauma to the spinal cord. The most dramatic clinical feature of this being the loss of sensation accompanied by motor paralysis with initial loss but gradual recovery of reflexes.

Spinal shock can be defined as depressed spinal reflexes caudal to the level of spinal cord injury (SCI) while those rostral to the SCI remain unaffected. Ditunno et al (2), proposed a four-phase model for spinal shock in 2004. Phase 1 (areflexia/hyporeflexia) is characterised by a complete loss or weakening of all reflexes below the SCI. This phase occurs from zero to twenty four hours post injury. Phase 2 (initial reflex return) lasts for one to three days, and is characterised by the return of some, but not all, reflexes below the SCI. Cutaneous reflexesBulbocavernosus (BC), AW, and Cremasteric $(\mathrm{CM})$ become stronger during this period. Typically the DTRs are still absent. The first reflexes to reappear are polysynaptic in nature, eg $\mathrm{BC}$, while monosynaptic reflexes eg deep tendon reflexes (DTRs) are not restored until Phase 3. Restoration of reflexes is not rostral to caudal as previously believed, but instead proceeds from polysynaptic to monosynaptic. Phases 3 (early hyper-reflexia [four days to one month]) is characterised by the return of DTRs. The ankle jerk (AJ) usually precedes the knee Jerk (KJ). The Babinski sign (BS) follows the recovery of the AJ very closely. Cutaneous reflexes (BC, $\mathrm{A} \mathrm{W}$, and $\mathrm{CM}$ ) typically have appeared by the end of this period and only $10 \%$ of delayed plantar responses (DPRs) will persist beyond a month. Despite these general trends, the timing of reflex return is variableeven after completeSCI where a uniform temporalcoursemightbeexpected.Autonomic function continues to evolve with improvement in vagally mediated bradyarrhythmias and hypotension. Autonomic dysreflexia can begin to emerge. Phase 4 (spasticity/hyper-reflexia[1-12months])ischaracterised by hyperreflexia, or abnormally strong reflexes usually produced with minimal stimulation. The DPR has disappeared in the majority of cases. Cutaneous reflexes, DTRs, and the BS become hyperactive and respond to minimal stimuli.

We believe our patient had spinal shock as evidenced by the quadriplegia. Respiratory complications after cervical spinal cord injury occur in $60 \%$ of patients. Thesinglemost significant determining factor is the severity of spinal cord injury (3).

Respiratory complications are greatest in cervical cord injuries in which the patient has lost intercostal function and abdominal muscle function needed to support a vigorous cough (4). The phrenic nerve is involved inlesions above $\mathrm{C}$ 5. In lesions below $\mathrm{C} 5$, with 
a functioning diaphragm and accessory muscles, and in the absence of lung injury or pre- existing disease, most patients can support satisfactory ventilation.

There is a tendency, even with good general care, for ventilatory function to deteriorate by the third or fourth day because of fatigue and retained secretions, and intubation and ventilation may be required early (5).

Pathophysiology: Uponsignificantspinalcord trauma the spinal cord may still appear grossly normal. The only microscopic abnormality may be central gray matter petechial haemorrhages with some extensions into the white matter (6). Reproducible changes in vascular integrity, blood flow, ionic shifts, and $\mathrm{pH}$ are recognised in the contusion model. Central spinal cord vessels incur endothelial damage, which results in platelet thrombosis, reduced blood flow, and ischaemia (7).

Microaneurysmal dilatations in gray matter arterioles may result in haemorrhage and further ischaemic damage (8). In contrast, spinal cord hyperemia has been reported by some researchers but this has usually been associated with incomplete injury and transient neurological deficit (9). Neuronal injury permits large sodium and potassium fluxes, which depolarise the membrane. Lactic acidosis results from decreased tissue oxygen and cellularATP, resulting in uncoupling of oxidative phosphorylation and a shift to anaerobic glycolysis (10).

Intracellular calcium mediates further cytotoxic events by activating proteases and phospholipases which break down myelin and damage axoplasm $(10,11)$. Release of arachidonic acid results in lipoperoxidation and oxygen free radical formation (12). This causes further membrane disruption and, thus, also arrests mitochondria and endoplasmic reticulum function. Free radicals also inhibit prostacyclin PGI which is an important inhibitor of platelet aggregation and thrombosis $(13,14)$.

Currently, a high dose methylprednisolone infusion is the only medical therapy that has been shown to influence outcome after spinal cord injury in humans. Possible mechanisms of this agent include protection of cell membranes against lipid peroxidation (10).

We postulate that the spinal canal stenosis occasioned by the multiple osteophytes and canal stenosis at C5, C6 level, combined with the minimal trauma, both potentially innocuous by themselves, have combined to resultin a potentially life threatening course through rapidly progressive spinal shock.

In conclusion, prompt recognition of this syndrome is of vital importance to prevent serious morbidity and possible mortality in patients with spinal shock. We pose questions that would warrant further research in this area. Should all patients with previous history of neck injury or cranio-vertebral anomalies who wish to be involved in contact sports, however minimal, have an MRI of their spine to rule out presence of spinal stenosis? Would spinal stenosis, if present, then be a contraindication to playing contact sports? Alternatively, should such individuals at risk, therefore, participate in contact sport only in a setup where early recognition and appropriate management facilities are immediately available?

\section{ACKNOWLEDGEMENT}

To the Aga Khan University Hospital, Nairobi for giving permission to publish this case.

\section{REFERENCES}

1. American Spinal Injury Association. International Standards for Neurological Classifications of Spinal Cord Injury. revised ed. Chicago, Ill: Amer. Spinal Injury Assoc. 2000; 1-23.

2. Ditunno, J.F., Little, J.W., Tessler, A. and Burns, A.S. Spinal shock revisited: a four-phase model. Spinal Cord. 2004; 42: 383-395.

3. Lemons, V.R. and Wagner, F.C. Respiratory complications after cervical spinal cord injury. Spine. 1994; 19: 2315.

4. Tator, C.H., Duncan, E.C. and Edmond, V.E. Complications and costs of management of acute spinal cord injury. Paraplegia. 1993; 31:700,

5. Hall, J. B., Smith, G.E. and Wood, L. D. H. Editors, McGraw-Hill. In: Principles of Critical Care, 3rd edition, 2005: 1409-1419.

6. Young, W. Blood flow, metabolic and neurophysiological mechanisms in spinal cord injury. In: Becker D.P., Povlishok J.T. (eds): CNS Trauma report. Bethesda, MD, National Institute for Neurological Disorders and Stroke, National Institutes of Health., 1985; 463- 473.

7. Nelson, E., Gertz, S.D. and Rennels, M.L. The role of vascular damage in the pathogenesis of central haemorrhagic necrosis. Arch. Neurol. 1977; 34:332333.

8. Collins, W.F., Piepmeier, J. and Ogle, E. The spinal cord injury problem-a review. Cent. Nerv. Syst. Trauma. 1986; 3:317-331.

9. Lohse, D.C., Senter, J.H. and Kauer, J.S. Spinal cord blood flow in transient paraplegia. J. Neurosurg. 1986; 18: $542-547$.

10. Janssen, L. and Hansebout, R.R. Pathogenesis of spinal cord injury and newer treatments. Spine. 1989; 14: 23-31.

11. Iwasaki, Y., Yamamoto, H. and lizuka, H. Supression of neurofilament degradation by protease inhibitors in experimental spinal cord injury. Brain Res. 1987; 406: 99-104.

12. Demopoulos, H.B., Flamm, E.S. and Pietronigro, D.D. The free radical pathology and microcirculation in major central nervous system disorders. Acta. Physiol. Scand. 1980; 492: 91-119.

13. Anderson, D.K., Means, E.D. and Waters, T.R. Spinal cord energy metabolism following compressive trauma to the feline cord. J. Neurosurg. 1980; 53: 375-380.

14. Hsu, S.Y., Halushka, P.V. and Hogan, E.L. Increased thromboxane level in experimental spinal cord injury. J. Neurol. Sci. 1986; 74: 829. 